\title{
Comparison of the Standard Floor Impact Sound with Living Impact Source by Subjective Evaluation
}

Park, Hyeon $\mathrm{Ku}^{*}$
Kim, Kyeong Mo**

Kim, Sun-Woo***

\begin{abstract}
In the previous test, the verification of the standard floor impact source was carried out comparing the physical characteristics with living impact sources. The result was appeared the validation of the standard impact source was very low because of differences of physical characteristics. This study aims to evaluate annoyance and loudness of standard impact source which is used for the measurement of floor impact sound, and to compare the annoyance and loudness of living impact sources which are produced in real life. The impact sources considered are tapping machine, tire and impact ball as standard sources, and nine real sources which were chosen from the existing researches. The result showed differences of annoyance and loudness between standard impact sources and living impact sources, which means the standard impact sources may rate the performance of floor system inappropriately. In the future, the rating method should be examined how the standard impact sources are similar with real sources in the point of rating the performance of floor system.
\end{abstract}

Keywords : Floor impact sound, standard impact source, living impact source

\section{Introduction}

Of the sound performances of a building, the performance regarding the floor impact sound is evaluated using a standard impact source, while the airborne sound is measured using a speaker or a real sound source. The equipment noise is also measured using the live sound of the source to evaluate the performance of the subject space of the building element. However, the floor impact sound differs in its level within the target space (receiving room) depending on the level of the actual source. This will make it impossible to measure the level of noise to evaluate the performance of the subject building. Therefore, it is now required to use a standard impact source to measure and evaluate the floor impact sound. [1, 2] ISO had specified only the lightweight impact source. But, recently, it also added specification for heavyweight sources, as well. We believe such a trend represent the increasing interest in the heavyweight impact sound all over the world.

Watters, for example, built several mock-ups of high-

* Main \& Corresponding Author, Research Professor, Chonnam National University（soundpark@cricmail.net)

** Ph.D. Candidate, Graduate School of Chonnam National University

*** Professor, School of Architecture, Chonnam National University heels and compared their spectrums with the tapping machine. And, he discovered that the results of the two different types of sound sources were different. [3] After that, a number of other studies were conducted to reveal that the standard sound sources and the living sound sources were not exactly the same. [4,5] Especially, a new impact source was developed in order to solve the problems with the heavy impact sources by Tachibana, whose newly developed source is now recognized as the new standard. [6] As such, the standard impact sources, which are of high importance in evaluating the insulation performance of the floor impact sound of a building, has been criticized for its lack of relevance in many preceding studies.

The purpose of this study, therefore, is to verify if they really reflect the properties of the living impact sounds in an actual residential environment, so that we can determine whether such a standard can be used for its purposes. As we discovered through a review of the existing literature that there are physical differences between the standard impact sources and the living sources, we tried to verify the relevance of the standard impact sources through a subjective evaluation process. [7] In many preceding studies, the standard impact sources were compared with the living impact sources. However, the number of studies which dominantly used the living impact sources of a 
residential environment was scarce, while many of them were focus on walking or running. Also, subjective evaluation method was used to establish the method of evaluation or set criteria, while the number of studies to evaluate the relevance of the standard impact sources was very small. The impact sources used in this study are the tapping machine, which is the standard lightweight impact source and the impact ball, which is the standard heavyweight impact source. As for the living impact sources, we examined 30 or more living sound sources in the preceding studies [13] and selected 9 of them considering their weight and the sound of impact when they fall to be compared with the standards.

\section{Test Method and Details}

\subsection{Sound Sources for the Test}

In order to compare the similarities with the standard impact sound sources, we selected the types of the actual sounds from a residential environment that would be suitable for a subjective evaluation, based on the results of literature reviews and actual observations. (Table 1) In case of living sound sources which are dropped from a height to make a sound by impact, the height of dropping were set at $20 \mathrm{~cm}, 60 \mathrm{~cm}$, and 100 $\mathrm{cm}$, respectively. In case of a jump, we reflected actual cases of jumps for the dropping level, which were, respectively, $20 \mathrm{~cm}$ and $40 \mathrm{~cm}$. The tapping machine was set for only one type of sound source without designating dropping heights for walking and running of a child. The bang machine and the impact ball were dropped at $85 \mathrm{~cm}$ and $100 \mathrm{~cm}$ above the floor. The measurement of the sound sources was carried out in conjunction with the subjective evaluation. The measurement of the background noise was taken during the daytime. In accordance with KS F 2810, we applied the noises at the center of the living room of the residence on the upper floor and measured the sound using 5 different locations on the lower floor using microphones. In order to ensure credibility of the test data, each sound source was dropped three times or more to pick up only the proper sounds for analysis. The sounds generated by people were repeatedly measured for many times to compare a large number of data sets.
Table 1. Specification of floor impact sound sources

\begin{tabular}{|c|c|c|c|}
\hline Source & Impact source & Dropping height $(\mathrm{cm})$ & Weight \\
\hline \multirow{9}{*}{ living } & Dry cell (D) & \multirow{3}{*}{$\begin{array}{c}\text { 20(D20, B20, G20), } \\
\text { 60(D60,B60,G60), } \\
100(\mathrm{D} 100, \mathrm{~B} 100, \mathrm{G} 100)\end{array}$} & $23.8 \mathrm{~g}$ \\
\hline & Baseball (B) & & $132.1 \mathrm{~g}$ \\
\hline & Golf ball $(\mathrm{G})$ & & $45.85 \mathrm{~g}$ \\
\hline & $\begin{array}{l}\text { Child running } \\
(\mathrm{CR}, 10 \mathrm{yr})\end{array}$ & - & $31 \mathrm{~kg}$ \\
\hline & $\begin{array}{l}\text { Child running in } \\
\text { place(CRP) }\end{array}$ & - & $31 \mathrm{~kg}$ \\
\hline & $\begin{array}{l}\text { Woman walking } \\
\text { (WW, } 32 \mathrm{yr} \text { ) }\end{array}$ & - & $52 \mathrm{~kg}$ \\
\hline & Child jumping (CJ) & $20(\mathrm{CJ} 20), 40(\mathrm{CJ} 40)$ & $31 \mathrm{~kg}$ \\
\hline & Man jumping (MJ) & 20(MJ20), 40(MJ40) & $68 \mathrm{~kg}$ \\
\hline & Woman jumping(WJ) & $20(\mathrm{WJ} 20), 40(\mathrm{WJ} 40)$ & $52 \mathrm{~kg}$ \\
\hline \multirow{3}{*}{ standard } & Tapping machine(TM) & - & - \\
\hline & Tire (Bang machine, BM) & $85 \mathrm{~cm}$ & - \\
\hline & Impact ball (IB) & $100 \mathrm{~cm}$ & - \\
\hline
\end{tabular}

\subsection{Field Experiment}

The targets of evaluation included 5 newly built spaces. 2 apartments were selected and the tests were conducted using 2 flat surfaces, while 1 dormitory room in the campus was also used for the test. The characteristics of the target apartment were as shown in Table 2.

Table 2. Composition of floor in the objective building

\begin{tabular}{|c|c|c|}
\hline Field & Composition of floor (mm) & $\begin{array}{c}\text { Reverse } \\
\mathrm{A}^{*}\end{array}$ \\
\hline \multirow{2}{*}{ GA-1 } & \multirow{4}{*}{$\begin{array}{c}\text { hard floor }(10)+\text { mortar }(40)+\text { lightweight } \\
\text { porous concrete }(50)+\text { vibration absorber }(20)+ \\
\text { slab }(180)\end{array}$} & $\mathrm{L}: 33$ \\
\hline & & $\mathrm{H}: 52$ \\
\hline \multirow{2}{*}{ GA-2 } & & $\mathrm{L}: 37$ \\
\hline & & $H: 53$ \\
\hline \multirow{2}{*}{ GI } & \multirow{2}{*}{$\begin{array}{c}\text { hard floor }(10)+\text { mortar }(40)+\text { lightweight } \\
\text { porous concrete }(50)+\text { vibration absorber }(20)+ \\
\text { slab }(210)\end{array}$} & $\mathrm{L}: 46$ \\
\hline & & $\mathrm{H}: 54$ \\
\hline \multirow{2}{*}{ HU-1 } & \multirow{4}{*}{$\begin{array}{l}\text { hard floor }(10)+\operatorname{mortar}(40)+\text { lightweight } \\
\text { porous concrete }(50)+\text { vibration absorber }(20)+ \\
\text { slab }(180)\end{array}$} & $\mathrm{L}: 42$ \\
\hline & & $\mathrm{H}: 57$ \\
\hline \multirow{2}{*}{ HU-2 } & & $\mathrm{L}: 45$ \\
\hline & & $\mathrm{H}: 61$ \\
\hline
\end{tabular}

* L : lightweight, $\mathrm{H}:$ heavyweight

\subsection{Subjective Evaluation}

In the preliminary study phase, we examined the impact, spectrum characteristics, and the level distribution of the impact source. And, it was confirmed that the standard impact sources and the living sources were different in their characteristics. In this study, we conducted subjective evalutions in order to verify the validity of the standard impact sources in depth and logically supplement the 
physical difference.

In the subjective evaluation, we generated the impact sounds at the field and had the subjects evaluate the loudness and annoyance of the sound they heard. The evaluation of the impact source in terms of the loudness and the annoyance were conducted with a residential facility where the construction was completed by the residents have not moved in yet. The test was conducted in the living room of the residential facility, and the sound heard at the lower floor was recorded after generating impact sounds on the upper floor. The responses were given using a 7 -points Likert scale on the answer sheet for the level of the loudness and the annoyance, for 'cannot hear at all (no annoyance at all)' was 1 point and 'very loud' (very annoying) was 7 points. The subjects were college students and graduate school students with no hearing problems. The details of the participants were as shown in Table 3.

The subjective evaluation was compared and analyzed with the physical characteristic the impact source. The level of the impact sound and its relationship with the loudness, the relationship between the impact sound level and annoyance, the relationship between the loudness and the annoyance were compared and analyzed for any differences between various types of impact sources.

Table 3. Detail of test subject

\begin{tabular}{c|c|c|c|c}
\hline Field & Male & Female & Age & $\begin{array}{c}\text { Average } \\
\text { age }\end{array}$ \\
\hline GA-1 & 4 & 6 & $19 \mathrm{yr}-39 \mathrm{yr}$ & 24.3 \\
\hline GA-2 & 4 & 6 & $19 \mathrm{yr}-39 \mathrm{yr}$ & 24.3 \\
\hline GI & 8 & 2 & $20 \mathrm{yr}-39 \mathrm{yr}$ & 26.0 \\
\hline HU-1 & 4 & 4 & $24 \mathrm{yr}-39 \mathrm{yr}$ & 28.5 \\
\hline HU-2 & 4 & 4 & $24 \mathrm{yr}-39 \mathrm{yr}$ & 28.5 \\
\hline
\end{tabular}

\section{Analysis of the Physical Characteristics and Subjective Responses}

\subsection{The physical characteristics of the impact source}

For the impacted source, the spectrum measured in the living room of GA-1 was as follow. The measurement of the impact source was, due to its characteristics conducted at the maximum sound pressure level. The tapping machine was used to evaluate Leq for reference in conjunction with it. Fig. 1 shows the result of the measurement by a lightweight impact source. For comparison, the base ball was dropped at $40 \mathrm{~cm}$ (Baseball_40.) The maximum sound level of the standard lightweight impact source, which is the tapping machine, was higher than those of the living impact sources at low frequency bands. But, it becomes relatively lower when the frequency band was around $500 \mathrm{~Hz}$. (Fig. 1). The standard heavyweight impact sources of tire (bang machine) and ball (impact ball) showed similar characteristics of the spectrums compared to the living impact sources. (Fig. 2 ). However, at the low frequency band, the level was much lower than those from the living impact sources, (such as a jump.) On the other hand, the level was higher than the living ones at higher frequency bands. Especially, tires and balls showed differences in their detailed frequency characteristics as they did in other studies. [4, 8]

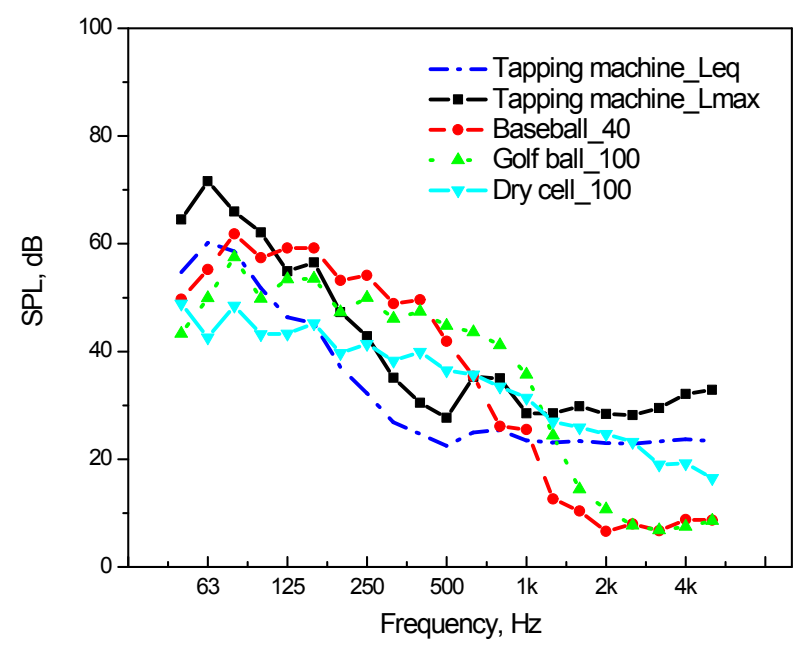

Fig. 1. Spectrum of lightweight impact sound measured in the field (excerpted from previous study [14])

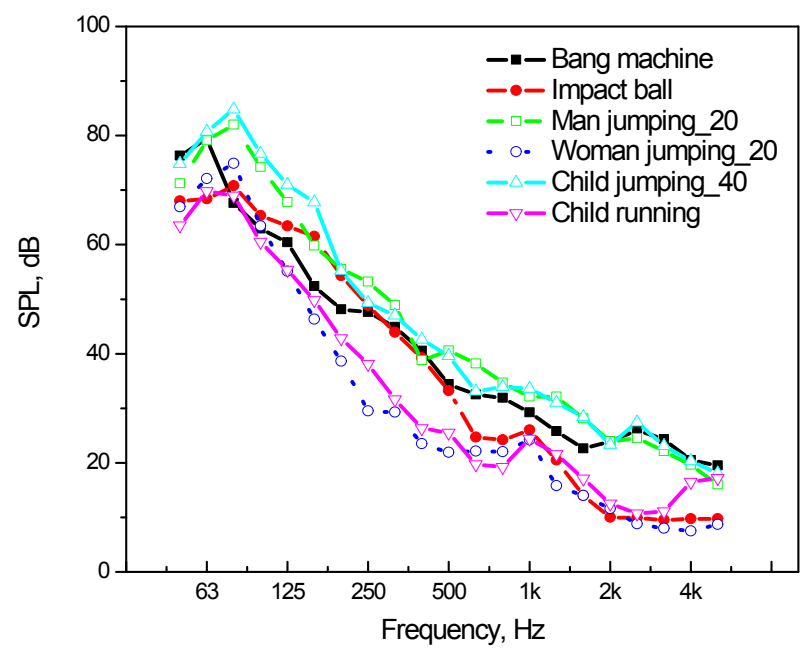

Fig. 2. Spectrum of heavyweight impact sound measured in the field (excerpted from previous study [14])

(1) Lightweight impact source

The max sound pressure levels measured in five different apartments were as shown in Fig. 3. As the layout and cross-sections, as well as other physical environment, were different, it is still possible that the level appear different for the same impact source. The 
dropping height of the impact source proportionally changes the level. But, the battery (D) showed relatively weaker proportional dependence to the dropping height. This is because the batteries may contact the floor with different parts of its surface when they impact the floor, and this makes irregular patterns which are not exactly proportional to the sound level. The standard impact source, that is, the tapping machine $(\mathrm{TM}$, showed similar levels with base ball (B) at $20 \mathrm{~cm}$ and with golf balls $(G)$ at $100 \mathrm{~cm}$. The living impact sources showed obvious differences in the sound pressure level for the same impact source depending on the conditions of the test environment. But, the standard impact source, that is, the tapping machine, showed very small differences. Such results make us question the ability of the tapping machines to measure the performance of the floor materials properly. The impact sound levels of $\mathrm{GA}-1$ and $\mathrm{GA}-2$, and those of $\mathrm{HU}-1$ and $\mathrm{HU}-2$, as well, were similar as those pairs were made of the same floor structures.

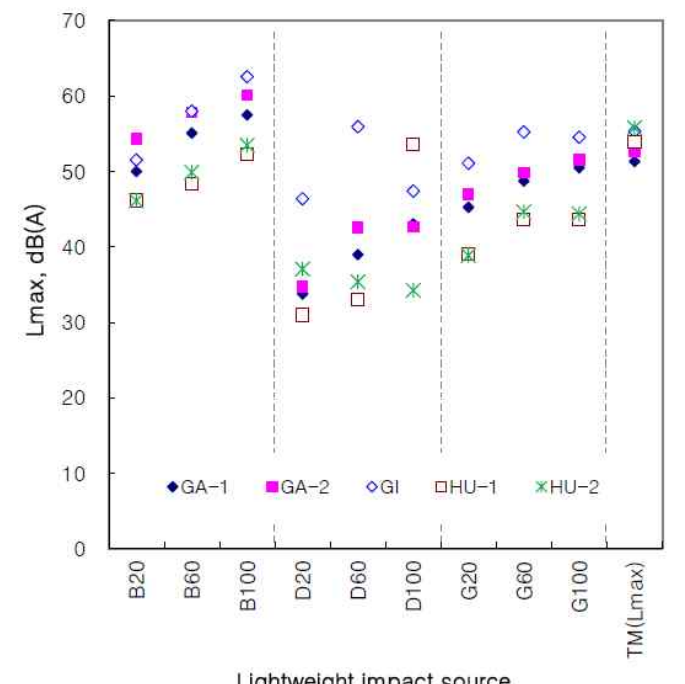

Fig. 3. Maximum sound level of lightweight impact sound(Lmax) (excerpted from previous study [14])

\section{(2) Heavyweight Impact Source}

Fig. 4 shows the comparison of the Lmax value for each place of measurement using heavy impact sources. The order of the sound pressure level for the same impact source is expected to be in proportion to the performance of the floor structure. But, the figure shows a mixed order without regard to the performance. Especially, while the lightweight impact sources showed higher relevance of the sound pressure level with the target building. But, for the heavyweight impact sources, the impact sound level depended on the types of sound sources rather than the structure of the building, resulting in a wide range of impact sound level. We believe this is because the level of impact is not standardized for the target buildings, the results lacked consistency. In spite of this, the fact that similar floor structures show similar impact sound levels is believed to be evidence showing that the impact forces in question are similar to one another. Also, as the purpose of this study is to see the relationship between the impact sound from an impact source and the subjective responses, further analysis would help us understand the relationship between the levels and the performance of the buildings through the relationships with the subjective responses.

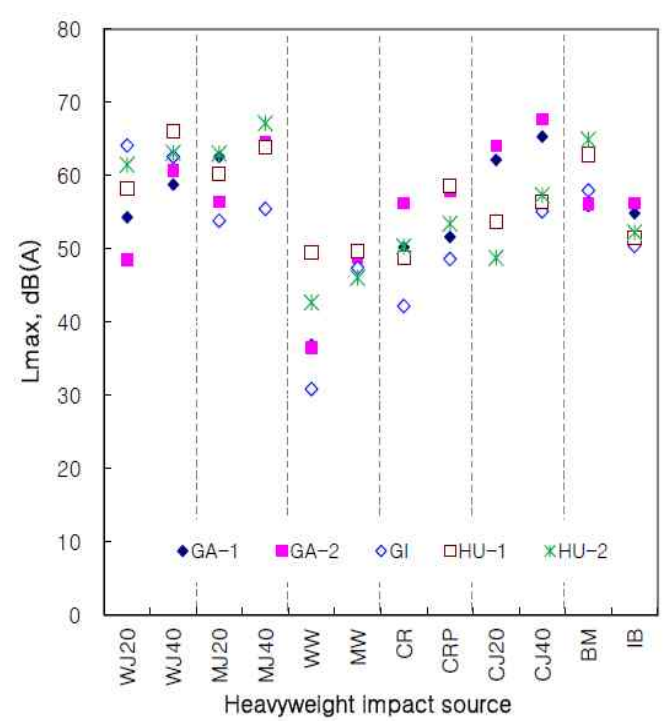

Fig. 4. Maximum sound level of heavyweight impact sound(Lmax) (excerpted from previous study [14])

\subsection{Results of Subjective Evaluation}

(1) Lightweight impact source

The physical indices of the impact sources were compared to one another. At the same time, we analyzed the resultant values of the subjective evaluation. Fig. 5 shows the evaluation results of the subjective evaluation. Compared to the standard impact sources, the differences in the subjective responses were larger in general. As one could expect the loudness and the annoyance showed similar patterns, while the values of the annoyance showed a wider distribution.

In case of the living impact sources, the reactions to the baseball (B) were the highest, while the response values toward the golf ball was relatively low compared to those of the baseball. The subjective responses to the dropping sound of a battery were all 4 or below, which was intermediately. This is thought to be very low compared to the physical indices we examined earlier. 
As for the tapping machine, which is the standard impact source, the physical indices were lower than those of the baseball at certain heights. However, the results from the subjective evaluation were higher than those from other impact sources, as one could see. Especially, the value of the annoyance was closing to 7 , which is even louder than those of the loudness. Especially, the tapping machine gave its characteristic, continual sounds, which are not likely to happen in real life. They were different from the single-timed impact sources, as well, which led to different subjective responses from others. With such results, we concluded that it is now necessary to analyze if the tapping machines measure the performance of the floor structure properly, because of its different subjective responses from those of the living impact sources.

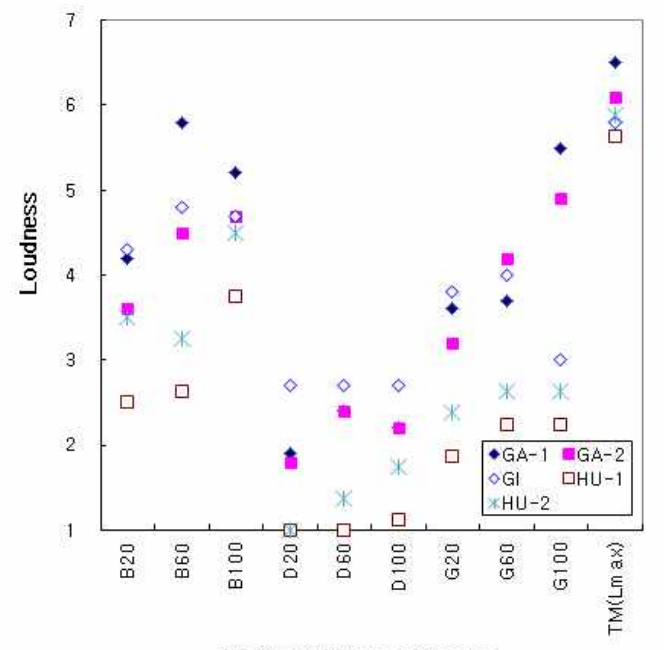

Lightweight impact source

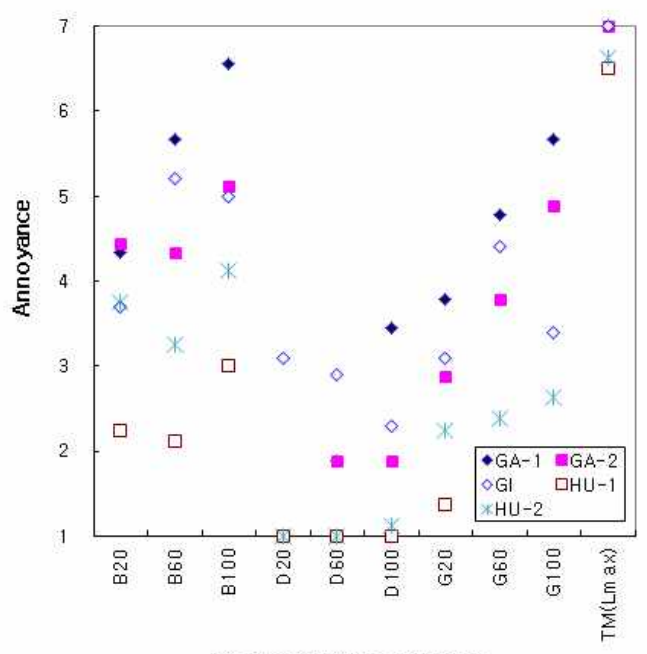

Lightweight impact source

Fig. 5. Loudness and annoyance for the lightweight impact sound (top : loudness, bottom : annoyance)
(2) Heavyweight Impact Source

The subjective responses for the heavyweight impact sources were somewhat different from those of the lightweight impact sources. Fig. 6 shows that, while the loudness and annoyance values of the lightweight impact sources were almost similar to each other, those of the heavyweight impact sources were different, where the annoyance value was much higher than the value of loudness. This means that the annoyance cause by the sound is actually larger than the level of loudness, making such sounds more annoying than those of light impact sources.

The subjective response for the living impact source was especially high with jumping. Followed by jumping of children, running, and walking. Especially, jumping of a grownup was very high (7) making it by far the loudest.

The subjective response value of the tire, which is a standard impact source, was similar to that of a jump by a grownup man. MJ4) and higher than other impact sources, as well. The impact ball (IB) were not as high as the living impact sounds from jumping, running, etc., in terms of the loudness. The annoyance value was also lower than other heavy impact sounds, except for walking.

The graph that compared the physical indices showed similar distributions between jumping and the tire. The impact ball was similar to the level of a child running. But, the subject responses to the tire were higher than the physical indices, while those of the impact ball were rather lower, instead. Especially, the loudness value, which shows the sense of loudness, also differed from those of the annoyance, meaning that it is questionable if the standard heavyweight impact sources can properly represent the living impact sources or not. The characteristics in the tires and the impact balls, which were the standard impact sources, are believe to need more reference items for the use of the recommendation for the sound sources and the use of the sound sources through the analysis if the evaluation method.

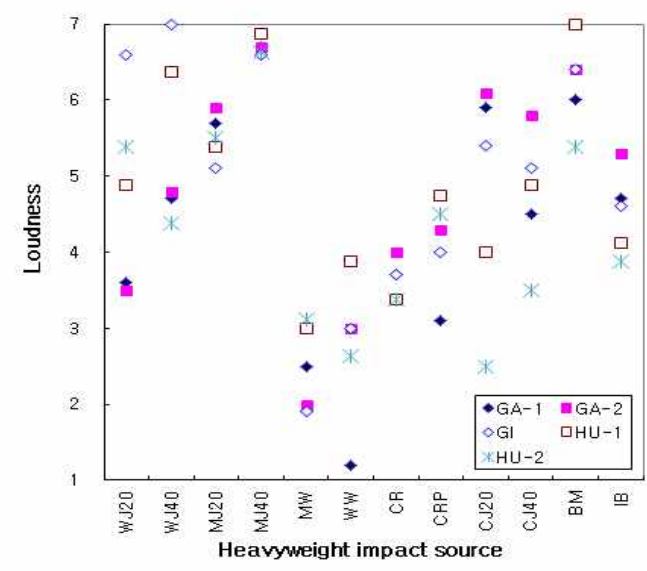




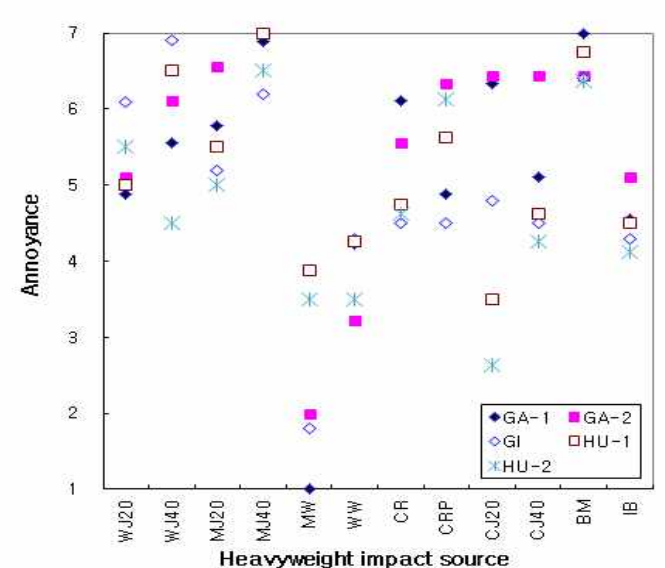

Fig. 6. Loudness and annoyance for the heavyweight impact sound (top : loudness, bottom : annoyance)

\section{Analysis and discussions}

\subsection{Sound pressure level and the loudness}

In order to analyze the relationship between the physical indices and the loudness that evaluated the level of the sound, the values of $\operatorname{Lmax}$ and $\mathrm{dB}(\mathrm{A})$ were used. Fig. 7 shows the relationship between the levels of the lightweight impact sources and the loudness, where it is necessary to see the relationship between the standard and the living impact sources and the distribution of the subjective responses evaluations with the regression line that is drawn straight.

The tapping machine, which is the standard impact source was discovered to be heard as much louder than the living impact source, the dropping sound of a battery was the lowest, while the reaction for the golf ball was the highest. Especially, compared to the trend line, the values of the tapping machine were too high, indicating that they are sensed as much louder for their actual loudness level.

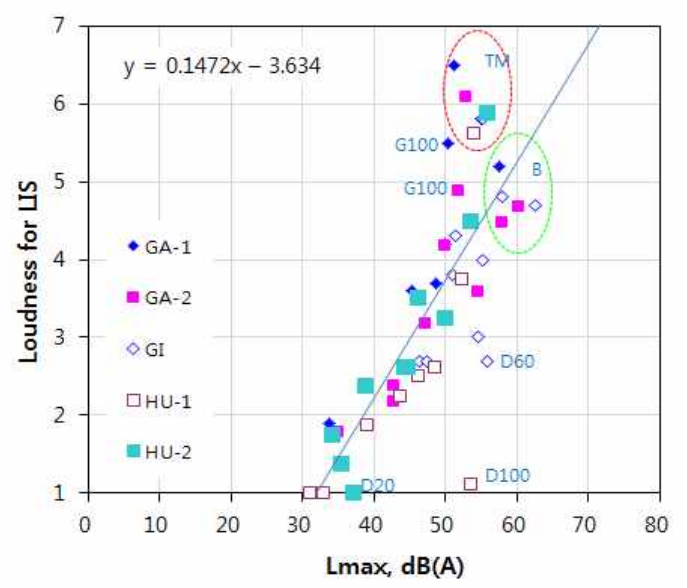

Fig. 7. Relationship between sound level and loudness for the lightweight impact sound
As for a heavyweight impact source, the standard impact sources of the tires and the impact ball were closer to the trend line that the sound from the lightweight impact sources. (Fig. 8). As for the tire, the level and the subjective responses were positioned above the trend line, meaning that they are sensed as louder than they actually are. The impact balls were positioned at around 4 to 5 of the sense of loudness, and closer to the trend line, meaning it well represents the living impact source.

In case of a jump by a grownup, which is a living impact source, it was evaluated much higher than it actually was and close to the trend line. In the meantime, the tire sounded not as loud as it actually did in terms of the sound level. Especially, in case of a jumping child, the measured level values were similar to those of a jumping adult. But, the subjective responses values were lower. Walking showed the lowest level of subjective responses, signifying that it would have very limited influence over the residents in real life. Especially, as we can see from the structure of the floors, the sense of loudness were different for the tire and the impact ball, which are the standard impact sources. It is needed to see this matter in conjunction with the performance evaluation of the floor structure.

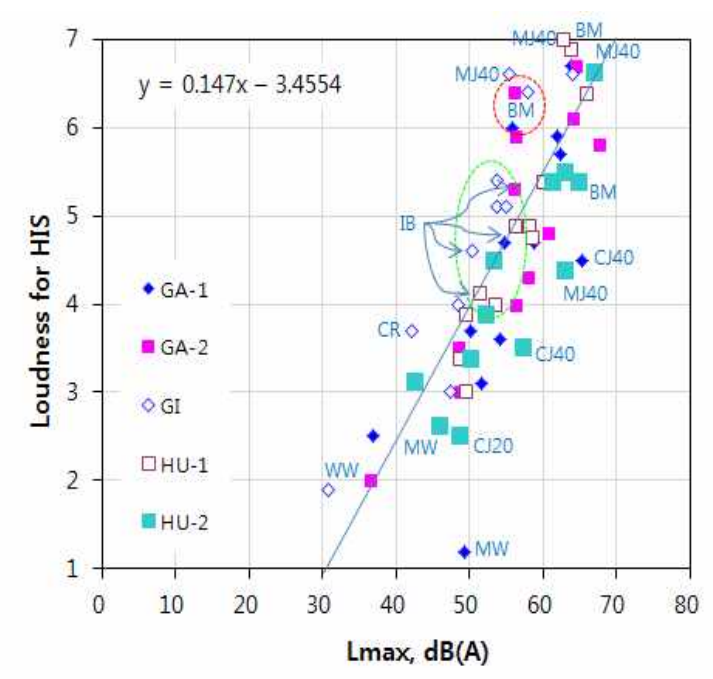

Fig. 8. Relationship between sound level and loudness for the heavyweight impact sound

\subsection{Sound pressure level and the annoyance}

The annoyance by the lightweight impact source showed a steeper inclination in terms of the changes of the sound pressure level when it was compared to those of the loudness. (fig. 9). This means that the sound from light impact sources annoys the subjects more for the actual level of sound pressure. 
The value of annoyance caused by the tapping machine deviated significantly from the regression line, meaning they are quite annoying. On the other hand, the living impact source of the base ball (B) was higher in the sound level compared to the tapping machine. But, the annoyance value for this sound was 5 or lower, which was very low indeed. Also, as for the golf ball, the level was lower than those of the base ball, but the annoyance level was similar. Such results are thought to be connected to the size of the impact source. Also, the features of the sound changes depending on the area of contact when the objects hit the floor, and it is believed that this difference is influencing the results of the subjective evaluations.

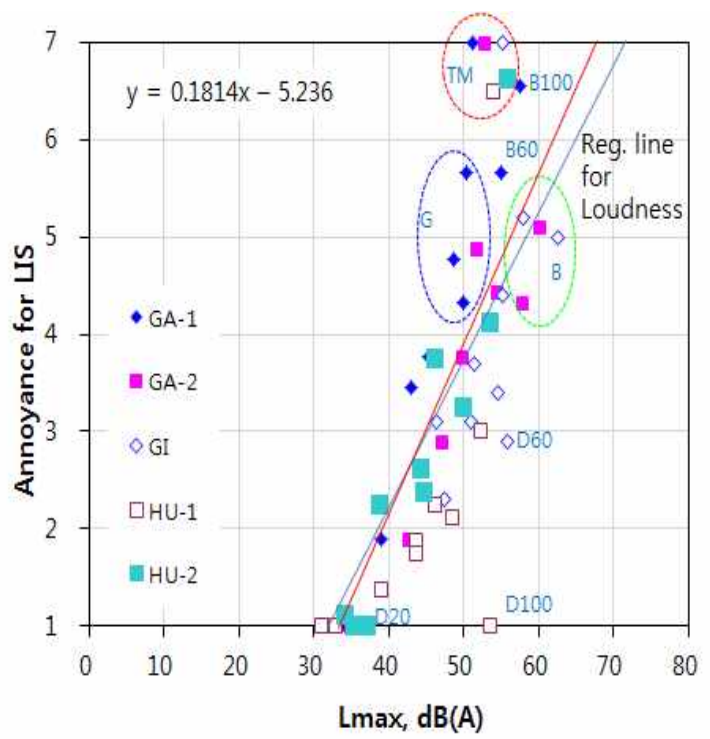

Fig. 9. Relationship between sound level and annoyance for the lightweight impact sound

For the heavyweight impact sources, the annoyance value for the different sound pressure level showed different relationships compared to those of the loudness. (Fig. 10). The tire, which is the standard impact source, was lower in terms of the loudness. But, one can see that it closes to 7 when it comes to the annoyance. A jump by an adult also shows very high annoyance values. The annoyance caused by the impact balls showed similar levels with those of the loudness. And, there were very closely distributed to the trend line, meaning they well represented the characteristics of a living impact source. However, it needs further monitoring to see how the fact that the level was not very high compared to that of the living impact source would affect the evaluation of the performance of the floor structures.

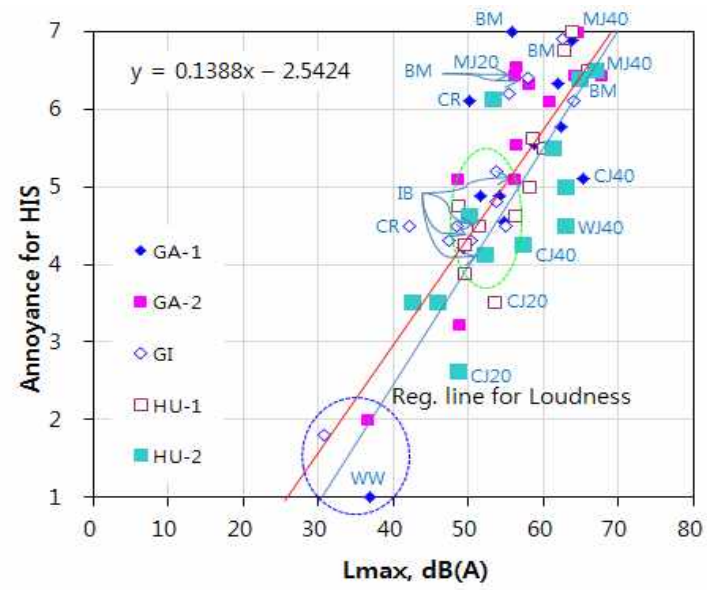

Fig. 10. Relationship between sound level and annoyance for the heavyweight impact sound

\subsection{The relationship between loudness and annoyance}

After we reviewed the physical indices and its relationship with the subjective responses, we could see that the loudness and the annoyance perceived for the same sound may differ. This may be attributable to the difference in the spectrums or the tone. But, since the purpose of this study is to compare the living impact sources and the standard impact sources, we examined and compared the characteristics of the standard impact sources with those of the living sources. The values of the tapping machine (lightweight standard) and the tire (heavyweight standard) were distributed higher than the trend line. The impact ball, on the other hand had its values very closely located to the trend line.

With this, we used the subject responses from the subjects to examine the relationship between the perceived loudness and the perceived annoyance. Fig. 11 shows that the loudness and the annoyance of the light impact sources are very closely related to one another. The loudness and annoyance of the tapping machine were the highest, followed by those from the base ball and the golf ball.

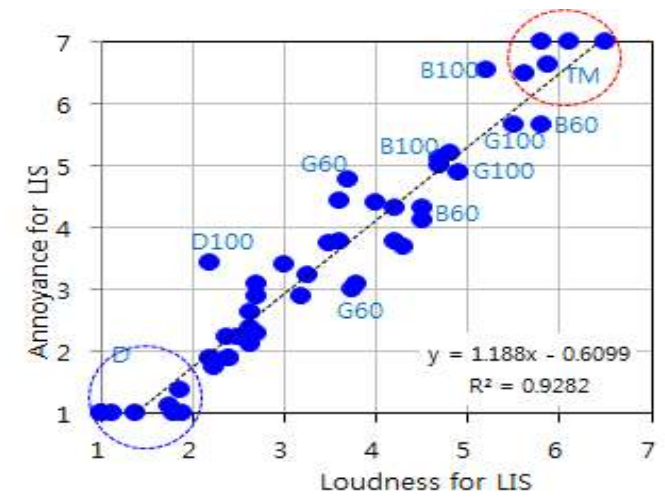

Fig. 11. Relationship between loudness and annoyance for the lightweight impact sound 
As one looks at the relationship with the heavy impact sources, as shown in Fig. 12, the extent of the correlation is significantly lower compared to that of the lightweight impact source. The tire showed higher level of annoyances compared to the level of the loudness. The impact balls, on the contrary, showed lower level of annoyances for the level of loudness. This shows that, as mentioned earlier, the performance of the floor structure is determined by the levels of the standard impact courses, so that it necessitates a verification process to make shore if the tire and the impact ball reflect he subjective responses well enough to evaluate the floor structure appropriately, or, which of the two sources have more differentiated results. In an existing study [15], it was shown that the evaluation value of the tire and the impact ball had different trends in accordance with the performance. Therefore, we believe it is necessary to widen the range of performance of the floor structure for evaluation to see how well the difference represents the results from the living impact sources.

As for the living impact sources, the adult jump, the female jump, and the child jump all had higher subjective response values. But, as they all show distributions that are very similar to the trend line, they show higher level of annoyance compared to that of children running. (CR, CRP) This indicates that the annoyance level is higher with continual noises caused by running than with the single-time noises by jumping.

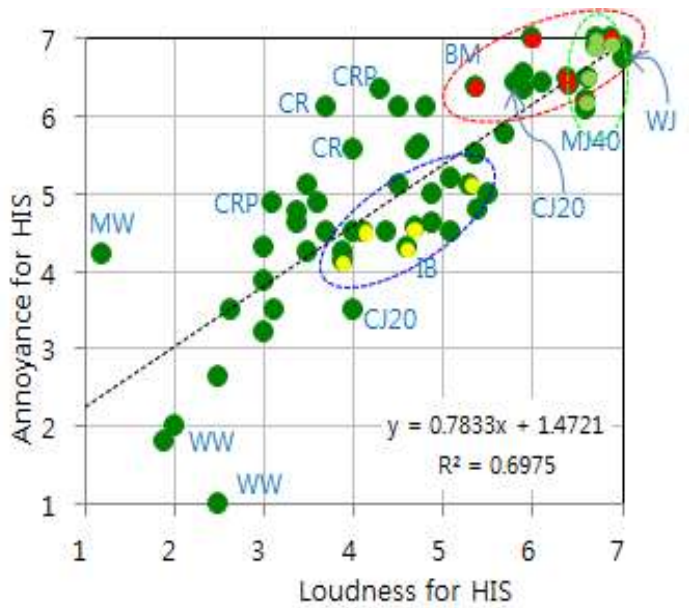

Fig. 12. Relationship between loudness and annoyance for the heavyweight impact sound

\subsection{Analysis of the relevance of the standard impact sources}

(1) Tapping Machine

Fig. 13 shows that the live impact showed different physical levels depending on the floor structure, and the subjective evaluation values were also different. But, the tapping machine, which was the standard impact source, had both of high levels and high subjective response values. The living impact sources showed significant differences depending on the structure. But, the fact that the standard sources showed a low level of difference means that its capability of comparing the structure is not as high as one might desire.

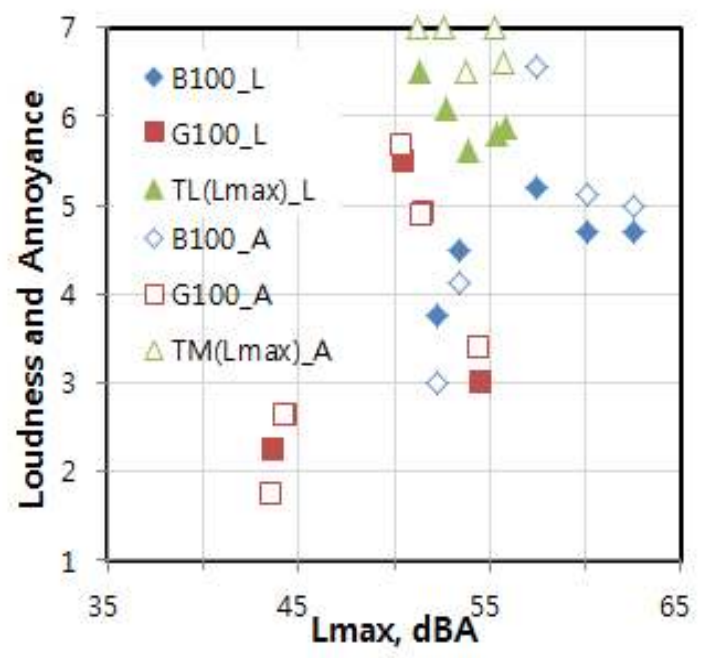

Fig. 13. Comparison of subjective response for the lightweight impact sound from tapping machine, golf ball and base ball

(2) Tire and Impact ball

In order to evaluate the performance of the floor structure, we analyzed the evaluation method using the subjective evaluation [16,17] The tire showed higher level of annoyance compared to the live sources. On the other hand, the impact ball showed low annoyance. The physical indices, too, were higher with the tire in terms of the impact. But, the impact ball's impact force was not higher compared to the living sources. A similar trend is also discovered in Jeon et al. [18]. As the standard for the floor impact sound was determined by the tire, which as the standard at that time, the impact ball should consider the corresponding difference. In addition, the reference value would have to be set to reflect the differences in the levels of loudness as well as the subjective responses between the two impact forces.

Especially, the range of the level and the subjective responses for the tire are lower than the difference between the physical indices and the subjective responses of the living impact sources. This means it is necessary to check if the standard can represent the living impact sources sufficiently with regard to the differentiation of the floor impact sound. Also, as the impact ball showed lower impact forces compared to those of the living impact sources, it would be necessary 
to check if they represent the live impact sources of higher impact forces.

(3) The necessity of verifying the relevance using the evaluation curve

When one establishes the standard and the evaluation method of the floor impact sound, the annoyance and the loudness of the impact sounds are to be evaluated and determined. [16, 17] However, as we have seen earlier, if the subjective responses are not in a linear correlation with the standard compared to the case with the living impact source, the standards set could either be higher or lower than the actual values. Also, it would be necessary to evaluate whether the testing methods in KS F 2863-2 sufficiently represent the characteristics of the living impact sources due to the same reason.

\section{Conclusion}

In this study, we analyzed whether the standard impact sources for floor impact sound measurement represent the living impact sources well enough by means of a subjective evaluation. The results showed that the standards were different from the living sources.

The standards had a narrower range of values for the test apartment, compared to the case with the living impact sources. To say that the range of measurement is small is that the range of differentiation of the performance can be narrower, making it necessary for us to further verify their capability to evaluate the differences properly. The results of the comparison between the sound pressure level and the subjective responses showed that the tapping machines showed higher loudness and annoyance compared to those of the living impact sources. The impact sound of the tire, too, showed higher loudness and annoyance compared to those of the actual heavyweight sources. On the other hand, the impact ball well represented the living source. With this fact, one can conclude that the impact ball better represent the living impact sources compared to the tires. However, the impact sound level of the impact balls was not higher than the sounds from living impact sources, such as jumping. Therefore, we could not determine whether it could be appropriate to use it for impact sounds of higher level.

The analysis of the relationship between the loudness and the annoyance of the target sound, accompanied by the comparison between the subjective responses and the linear trend, showed that the tapping machine and the tire had higher annoyance compared to the loudness, and the impact ball was even lower. This could bring about some changes in the testing methods. Especially if we consider the current status that tire and impact ball are used as heavyweight impact sources, further studies would be necessary to validate the standard values and the measurement methods.

In this study, the subjective evaluation was performed for the living impact sounds at the field to compare the differences between the standard and the living impact sources. Therefore, this study has its limitations in quantifying the relevance of the standard sources. For this reason, further studies would be necessary to analyze the quantifiable differences between the standard and the living impact sources and suggest an appropriate method of comparing them.

\section{Acknowledgement}

This research was supported by Basic Science Research Program through the National Research Foundation of Korea(NRF) funded by the Ministry of Education, Science and Technology(2012006695, No. 2005-0049719).

\section{References}

[1] KS F 2810-2:2012, Field measurements of floor impact sound imsulation of buildings - Part2 : Method using standard heavy impact sources, 2012

[2] ISO 10140-3, Acoustics - Laboratory measurement of sound insulation of building elements - Part 3: Measurement of impact sound insulation, 2010.9

[3] B. G. Watters, Impact-Noise Characteristics of Female HardHeeled Foot Traffic, J. Acoust. Soc. Am., 37(4), 1965

[4] Wanqing Shi et. al., An Investigation of the Characteristics of Impact Sound Sources for Impact Sound Insulation Measurement, Applied Acoustics, 51(1), 1997

[5] D. Olynyk et. al, Assessment of Footstep Noise through Wood-Joist and Concrete Floors, J. Acoust. Soc. Am., 43(4), 1968

[6] H. Tachibana et. al., Development of new heavy and soft impact impact source for the assessment floor impact sound of building, Proceedings of Inter-noise 98, 1998, ISO 10140-3

[7] Park et. al., Subjective evaluation for the floor impact sound by standard and living impact sources, proc. of the Korean Society for Noise and Vibration, 2010.10

[8] Jeon et. al., Comparison of standard floor impact sources with a Human impact source, Transactions of the Korean Society for Noise and Vibration, 16(8), 2006,

[9] Jeon et. al., Subjective evaluation of floor impact noise generated by children and standard impact machine, J. of Architectural Institute of Korea, 18(1), 2002.01

[10] Kim S. W. et. al., A study on the standard and classification of impact sound insulation for heavy wight source in apartment house, J. of Architectural Institute of Korea, 9(7), 1993.07, 
[11] E. Gerretsen, A new system for rating impact sound insulation, Applied Acoustics, 9(4), 1976,

[12] K. Bodlund, Alternative reference curves for evaluation of the impact sound insulation between dwellings, J. Sound and Vibration, 102(3), 1985

[13] H. K. Park et. al., Comparing living floor impact sound with the standard floor impact source in apartment, proceeding of ICSV16, 2009.

[14] Park H. K. et. al., Verification of effectiveness of the standard floor impact source by comparing with living impact sources, Transactions of the Korean Society for Noise and Vibration, 23(12), 2013.

[15] Shin H. et. al., A Study of Rating Method Comparison for Heavy-weight Floor Impact Sound based on the Field Test Data in Apartment Houses, J. of Korea Institute of Ecological Architecture and Environment, 10(5), 2010.

[16] Song M. J. et. al., A study on the sound classification system for floor sound insulation performance of apartment houses, J. of Architectural Institute of Korea, 20(10), 2004.10.

[17] J. Ryu et. al., Subjective ratings of floor impact sounds in wood frame construction, inter-noise, 2009

[18] J. Y. Jeon et. al., Review of the Impact Ball in Evaluating Floor Impact Sound, Acta Acustica United with Acustica, 92, 2006

Received January 6, 2014;

Final revision received February 6, 2014;

Accepted February 10 2014; 